\title{
Perfect powers in arithmetic progression. A note on the inhomogeneous case
}

\author{
by
}

L. Hajdu (Debrecen)

Dedicated to Professor R. Tijdeman on the occasion of his sixtieth birthday

1. Introduction. Arithmetic progressions consisting of almost perfect powers are widely investigated in the "homogeneous" case. That is, one is interested in arithmetic progressions of the shape

$$
a_{0} x_{0}^{l}, \ldots, a_{k-1} x_{k-1}^{l} \quad \text { with } \quad a_{i}, x_{i} \in \mathbb{Z}(0 \leq i \leq k-1),
$$

with some fixed integer $l \geq 2$, such that the coefficients $a_{i}$ are "restricted" in some sense. It was known already by Fermat and proved by Euler (see [D, pp. 440 and 635]) that four distinct squares cannot form an arithmetic progression. The contributions of Darmon and Merel [DM] on the Fermat equation imply that there are no three $l$ th powers with $l \geq 3$ in arithmetic progression, up to the trivial cases.

In this paper we take up the problem when the arithmetic progression consists of almost perfect "inhomogeneous" powers. Let $S=\left\{p_{1}, \ldots, p_{s}\right\}$ be any set of positive primes with $p_{1}<\cdots<p_{s}$, and write $\mathbb{Z}_{S}$ for the set of those non-zero integers whose prime divisors belong to $S$. Put

$$
H=\left\{\eta x^{l} \mid \eta \in \mathbb{Z}_{S}, x, l \in \mathbb{Z} \text { with } x \neq 0 \text { and } l \geq 2\right\},
$$

and note that $\pm 1 \in H$, but $0 \notin H$. To guarantee that the representation of every element $h \in H$ is unique, we further assume that for $h=\eta x^{l}$ the number $\eta$ is $l$ th power free, $x>0$, and $l=2$ if $h \in \mathbb{Z}_{S}$. In particular, if $x=1$ then $\eta$ is square-free. The main purpose of this paper is to show that the $a b c$ conjecture implies that the number of terms of any "coprime"

2000 Mathematics Subject Classification: Primary 11D41.

Research supported in part by the Netherlands Organization for Scientific Research (NWO), by grants T42985 and F34981 of the Hungarian National Foundation for Scientific Research, and by the FKFP grant 3272-13/066/2001. 
arithmetic progression in $H$ is bounded by a constant $c(s, P)$ depending only on $s=|S|$ and $P=p_{s}$. Moreover, the number of such progressions having at least three terms, where the exponents of the powers are $\geq 4$, is finite. We derive a similar statement unconditionally, provided that the exponents of the terms in the progression are bounded from above. Our main tools, besides the $a b c$ conjecture, will be a theorem of Euler on equation (1) below with $l=2$, the above mentioned result of Darmon and Merel on Fermat-type ternary equations, and a famous theorem of van der Waerden from Ramsey theory, about arithmetic progressions.

Finally, we mention that our problem is related to the equation

$$
n(n+d) \cdots(n+(k-1) d)=b y^{l}
$$

in non-zero integers $n, d, b, y, k \geq 2, l \geq 2$ with $\operatorname{gcd}(n, d)=1, P(b) \leq k$, where for any integer $u$ with $|u|>1$ we write $P(u)$ for the greatest prime factor of $u$ and we put $P( \pm 1)=1$. It is easy to show that using (1) one can write

$$
n+i d=a_{i} x_{i}^{l} \quad \text { with } \quad P\left(a_{i}\right) \leq k-1(0 \leq i \leq k-1) .
$$

Equation (1) and its various specializations have a very extensive literature. For related results we just refer to the survey papers and recent articles $[\mathrm{BGyH}],[\mathrm{Gy}],[\mathrm{GyHS}],[\mathrm{SS}],[\mathrm{S} 1]-[\mathrm{S} 3],[\mathrm{T} 1],[\mathrm{T} 2]$, and the references given there. We only mention two particular theorems about (1), which are relevant from our viewpoint. Shorey (see [S1]) proved that the $a b c$ conjecture implies that with $l \geq 4, k$ is bounded by an absolute constant in (1). Extending this result, Györy, Hajdu and Saradha [GyHS] deduced from the $a b c$ conjecture that with $l \geq 4$ and $k \geq 3$, equation (1) has only finitely many solutions. Thus our theorems yield a kind of extension of the above mentioned results of Shorey [S1] and Györy, Hajdu and Saradha [GyHS] to the inhomogeneous case. However, it is important to note that as $P\left(a_{i}\right) \leq k-1$ in (2), and we fix the prime divisors of the $l$ th power free part of $h \in H$ in advance, the results obtained here do not imply the corresponding theorems in $[\mathrm{S} 1]$ and $[\mathrm{GyHS}]$.

2. Main results. In what follows, $c_{0}, \ldots, c_{15}$ will denote constants depending only on $s$ and $P$. Though $s \leq P$, our arguments will be more clear if we indicate the dependence also upon $s$. By a non-constant arithmetic progression we will simply mean a progression with non-zero common difference.

TheOrem 1. Suppose that the abc conjecture is valid. Let $h_{0}, \ldots, h_{k-1}$ be any non-constant arithmetic progression in $H$, with $h_{i}=\eta_{i} x_{i}^{l_{i}}(0 \leq i \leq$ $k-1)$, such that $\operatorname{gcd}\left(h_{0}, h_{1}\right) \leq c_{0}$ for some $c_{0}$. Then

$$
\max (k, l)<c_{1} \text {, where } l=\max _{0 \leq i \leq k-1} l_{i} .
$$


Moreover, the number of such progressions with $k \geq 3$ and $l_{i} \geq 4$, is bounded by some $c_{2}$.

REMARK 1. Inspecting the proof of Theorem 1, one can easily see that the second part of the statement can be extended as follows. Consider progressions $h_{0}, \ldots, h_{k-1}$ as above, such that $k \geq 3$ and for all $i \in\{0, \ldots, k-1\}$ there exist $j, t \in\{0, \ldots, k-1\} \backslash\{i\}$ with $j \neq t$ and $1 / l_{i}+1 / l_{j}+1 / l_{t}<1$. Then the $a b c$ conjecture implies that the number of such progressions is bounded by some $c_{2}$.

Remark 2. The condition $\operatorname{gcd}\left(h_{0}, h_{1}\right) \leq c_{0}$ in Theorem 1 is necessary. Indeed, there exist non-constant arithmetic progressions in $H$ consisting of non-zero perfect powers, having arbitrarily many terms. To see this, observe that each pair of distinct positive perfect powers can be considered as a non-constant arithmetic progression of two terms. Suppose that for some $i \geq 2, h_{0}, \ldots, h_{i-1}$ is such a progression of positive perfect powers, say $h_{j}=x_{j}^{l_{j}}$ with $x_{j} \geq 1$ and $l_{j} \geq 2(0 \leq j \leq i-1)$. Let $t=2 h_{i-1}-h_{i-2}$ and $l_{i}^{\prime}=\prod_{j=0}^{i-1} l_{j}$, and write

$$
h_{j}^{\prime}=t^{l_{i}^{\prime}} h_{j} \quad \text { for } 0 \leq j \leq i-1, \quad h_{i}^{\prime}=t^{l_{i}^{\prime}+1} .
$$

In this way we obtain a non-constant arithmetic progression $h_{0}^{\prime}, \ldots, h_{i-1}^{\prime}, h_{i}^{\prime}$ consisting of positive perfect powers, having exponents $l_{0}, \ldots, l_{i-1}, l_{i}=l_{i}^{\prime}+1$. This verifies our claim, which shows that the assumption $\operatorname{gcd}\left(h_{0}, h_{1}\right) \leq c_{0}$ cannot be omitted.

If we drop the $a b c$ conjecture, we need a further assumption to get a finiteness statement for the number of terms in our arithmetic progressions.

Theorem 2. Let $l$ be a fixed integer with $l \geq 2$. Then for any nonconstant arithmetic progression $h_{0}, \ldots, h_{k-1}$ in $H$ such that $l_{i} \leq l$ in the representation $h_{i}=\eta_{i} x_{i}^{l_{i}}(0 \leq i \leq k-1)$, we have $k \leq C_{0}(s, P, l)$, where $C_{0}(s, P, l)$ is a constant depending only on $s, P$ and $l$.

REMARK 3. Note that in Theorem 2 we do not need the assumption $\operatorname{gcd}\left(h_{0}, h_{1}\right) \leq c_{0}$. However, the example in Remark 2 shows that the condition $l_{i} \leq l(0 \leq i \leq k-1)$ is necessary in this case.

Finally, we propose the following

CONJECTURE. Theorem 1 is true unconditionally, i.e. independently of the $a b c$ conjecture.

3. Some lemmas. To prove our theorems, we need several lemmas. The first one concerns almost perfect squares in arithmetic progression.

LEMma 1. The product of four consecutive terms in a non-constant positive arithmetic progression is never a square. 
Proof. This is a classical result of Euler (cf. [M, p. 21]).

Our next lemma is about Fermat-type ternary equations.

Lemma 2. Let $l \geq 3$ be an integer. Then the equation

$$
X^{l}+Y^{l}=2 Z^{l}
$$

has no solution in coprime non-zero integers $X, Y, Z$ with $X Y Z \neq \pm 1$.

Proof. This was proved by Darmon and Merel [DM].

The next lemma is from Ramsey theory, concerning arithmetic progressions.

Lemma 3. For any positive integers $u$ and $v$ there exists a positive integer $w$ such that for any coloring of the set $\{1, \ldots, w\}$ using $u$ colors, we get a non-constant monochromatic arithmetic progression, having at least $v$ terms.

Proof. This nice result is due to van der Waerden (cf. [vdW]).

The next statement yields the assertion of Theorem 1 unconditionally in case of homogeneous powers.

Lemma 4. Let $l$ be a fixed integer with $l \geq 2$. Suppose that $h_{0}, \ldots, h_{k-1}$ is an arithmetic progression in $H$ such that $h_{i}=\eta_{i} x_{i}^{l}$ for all $i=0, \ldots, k-1$. Then $k<C_{1}(s, P, l)$, where $C_{1}(s, P, l)$ is a constant depending only on $s, P$ and $l$.

Proof. Color the terms of the arithmetic progression $h_{0}, \ldots, h_{k-1}$ in such a way that $h_{i}$ and $h_{j}$ have the same color if and only if $\eta_{i}=\eta_{j}(0 \leq i, j \leq$ $k-1$ ). As $\eta_{i}$ and $\eta_{j}$ are $l$ th power free, at most $2 l^{s}$ colors are necessary. (We need the factor 2 because of the signs.)

Assume first that $l=2$. We apply Lemma 3 with $(u, v)=\left(2^{s+1}, 4\right)$ to conclude that if $k \geq w$ with some $w=w(s)$, then there exist $0 \leq i_{1}<$ $i_{2}<i_{3}<i_{4} \leq k-1$ such that $h_{i_{1}}, h_{i_{2}}, h_{i_{3}}, h_{i_{4}}$ is a non-constant arithmetic progression of non-zero integers, with $\eta_{i_{1}}=\eta_{i_{2}}=\eta_{i_{3}}=\eta_{i_{4}}$. Then we have

$$
h_{i_{1}} h_{i_{2}} h_{i_{3}} h_{i_{4}}=\left(\eta_{i_{1}}^{2} x_{i_{1}} x_{i_{2}} x_{i_{3}} x_{i_{4}}\right)^{2} .
$$

However, by Lemma 1, this is impossible. (Note that it does not make a difference whether $\eta_{i_{1}}$ is positive or negative.) This gives a contradiction, whence $k<w$, and the lemma follows when $l=2$.

Suppose now that $l \geq 3$. We apply again Lemma 3 , this time with $(u, v)=$ $\left(2 l^{s}, 3\right)$ to derive that if $k \geq w$ with some $w=w(s, l)$, then there exist $0 \leq i_{1}<i_{2}<i_{3} \leq k-1$ such that $h_{i_{1}}, h_{i_{2}}, h_{i_{3}}$ is an arithmetic progression with $\eta_{i_{1}}=\eta_{i_{2}}=\eta_{i_{3}}$. Hence we obtain

$$
x_{i_{1}}^{l}+x_{i_{3}}^{l}=2 x_{i_{2}}^{l} .
$$


By Lemma 2 , as $h_{i_{j}} \neq 0(j=1,2,3)$ and our progression is non-constant, we deduce that (3) is impossible. Thus we get a contradiction, whence $k<w$, and the lemma is proved.

REMARK 4. Note that assuming the $a b c$ conjecture, this lemma follows from the aforementioned result of Shorey [S1] in the case of $\operatorname{gcd}\left(h_{0}, h_{1}\right)=1$.

Lemma 5. Suppose that the abc conjecture is valid, and let $c_{3}=C_{1}(s, P, 2)$ be the constant given in Lemma 4 , corresponding to the exponent $l=2$. Then there exists a $c_{4}$ such that if $h_{0}, \ldots, h_{k-1}$ is any arithmetic progression in $H$ with $h_{i}=\eta_{i} x_{i}^{l_{i}}$ such that $\operatorname{gcd}\left(h_{0}, h_{1}\right)<c_{5}$ and $k \geq 2 c_{3}$, then $l_{i}<c_{4}$ for all $i=0, \ldots, k-1$.

Proof. Suppose that we have an arithmetic progression $h_{0}, \ldots, h_{k-1}$ as above, and take any $i \in\{0, \ldots, k-1\}$ with $l_{i} \geq 7$. (If no such $i$ exists, then the lemma follows with $c_{4}=7$.) Note that $x_{i}>1$. By Lemma 4 we infer that there exists a $j$ with $0<|i-j| \leq c_{3}$ such that $l_{j} \geq 3$. Choose any $t \in\{0, \ldots, k-1\} \backslash\{i, j\}$ with $|i-t| \leq 2$. Then for some coprime non-zero integers $\lambda_{i}, \lambda_{j}, \lambda_{t}$ with $\max \left(\lambda_{i}, \lambda_{j}, \lambda_{t}\right) \leq|i-j|+2$ we have $\lambda_{i} h_{i}+\lambda_{j} h_{j}+\lambda_{t} h_{t}=0$. This gives

$$
\lambda_{i} \eta_{i} x_{i}^{l_{i}}+\lambda_{j} \eta_{j} x_{j}^{l_{j}}+\lambda_{t} \eta_{t} x_{t}^{l_{t}}=0 .
$$

Let $D$ denote the gcd of the above three terms; observe that as $\operatorname{gcd}\left(h_{0}, h_{1}\right)$ $\leq c_{5}$, we have $D<c_{6}$.

We show that the $a b c$ conjecture implies that $l_{i}$ is bounded. Note that when $D=1$, and the coefficients of $x_{i}^{l_{i}}, x_{j}^{l_{j}}, x_{t}^{l_{t}}$ are fixed, by a similar argument Tijdeman derived from the $a b c$ conjecture that (4) has only finitely many solutions (see [T1, p. 234]). Let $r \in\{i, j, t\}$ be the index for which $\left|\lambda_{r} \eta_{r} x_{r}^{l_{r}}\right|$ is maximal among these three terms. The (effective version of the) $a b c$ conjecture with $\varepsilon=1 / 42$ gives

$$
\left|\lambda_{r} \eta_{r} x_{r}^{l_{r}}\right|<c_{7}\left(\prod_{p \mid x_{i} x_{j} x_{t}} p\right)^{43 / 42} .
$$

As $l_{i} \geq 7, l_{j} \geq 3$, and $l_{t} \geq 2$, whence $1 / l_{i}+1 / l_{j}+1 / l_{t}<1-1 / 42$, this yields

$$
\left|\lambda_{r} \eta_{r} x_{r}^{l_{r}}\right| \leq c_{8} x_{r}^{(1763 / 1764) l_{r}} .
$$

If $x_{r}=1$ (implying that $r=t, l_{r}=2$, and $\eta_{r}$ is square-free), then since

$$
x_{i}^{l_{i}}<\left|\lambda_{i} \eta_{i} x_{i}^{l_{i}}\right| \leq\left|\lambda_{r} \eta_{r} x_{r}^{l_{r}}\right|
$$

and $x_{i}>1$, we get $l_{i}<c_{9}$. Otherwise, $x_{r}>1$ gives $l_{r}<c_{10}$, whence $\left|\lambda_{r} \eta_{r} x_{r}^{l_{r}}\right|<c_{11}$. Thus using again (5) and $x_{i}>1$, we obtain $l_{i}<c_{12}$ also in this case. As $i$ was taken arbitrarily with $l_{i} \geq 7$, the statement follows with $c_{4}=\max \left(7, c_{9}, c_{12}\right)$. 
4. Proofs of the theorems. Now we are ready to prove our main results. It is more convenient to start with the proof of Theorem 2.

Proof of Theorem 2. Let $C_{2}(s, P, l)$ be the maximum of the values $C_{1}(s, P, L)$ defined in Lemma 4 , where $L$ ranges through the interval $[2, l]$. Apply Lemma 3 to our progression with $(u, v)=\left(l-1, C_{2}(s, P, l)\right)$. (The terms with the same exponents have the same colors.) Thus Lemma 3 gives some constant $C_{0}(s, P, l)$, depending only on $s, P$ and $l$, such that $k \geq C_{0}(s, P, l)$ would be a contradiction by Lemma 4 . Thus $k<C_{0}(s, P, l)$, and the theorem follows.

Proof of Theorem 1. We may suppose that $k \geq 2 c_{3}$, where $c_{3} \geq 2$ is given in Lemma 5 . Then by Lemma 5 we have $l_{i} \leq c_{4}$ for all $i=0, \ldots, k-1$. Thus the first part of the theorem follows from Theorem 2, with $c_{1}=$ $\max \left(c_{4}, C_{0}\left(s, P, c_{4}\right)\right)$.

To prove the second part, suppose that $l_{i} \geq 4$ for all $i=0, \ldots, k-1$. We already know that $\max (k, l)<c_{1}$. Fix $k$ and choose any distinct $i, j, t \in$ $\{0, \ldots, k-1\}$. Just as in the proof of Lemma 5 , we get an equation of the form

$$
\lambda_{i} \eta_{i} x_{i}^{l_{i}}+\lambda_{j} \eta_{j} x_{j}^{l_{j}}+\lambda_{t} \eta_{t} x_{t}^{l_{t}}=0
$$

with some integers $\lambda_{i}, \lambda_{j}, \lambda_{t}$ such that $\max \left(\left|\lambda_{i}\right|,\left|\lambda_{j}\right|,\left|\lambda_{t}\right|\right)<k<c_{1}$. Moreover, the gcd of the three terms on the left hand side is bounded by some $c_{13}$. Following the argument of Lemma 5 , as $x_{i}, x_{j}, x_{t}$ are all $>1$, and $1 / l_{i}+1 / l_{j}+$ $1 / l_{t} \leq 3 / 4$, using the $a b c$ conjecture we derive that $\max \left(x_{i}^{l_{i}}, x_{j}^{l_{j}}, x_{t}^{l_{t}}\right)<c_{14}$. As also $\max \left(\left|\eta_{i}\right|,\left|\eta_{j}\right|,\left|\eta_{t}\right|\right)<c_{15}$, the theorem follows.

Acknowledgements. The author is grateful to Cs. Sándor for his motivating question, and to the referee for his helpful and useful remarks.

\section{References}

[BGyH] M. A. Bennett, K. Győry and L. Hajdu, Powers from products of consecutive terms in arithmetic progression, J. Reine Angew. Math., submitted.

[DM] H. Darmon and L. Merel, Winding quotients and some variants of Fermat's Last Theorem, ibid. 490 (1997), 81-100.

[D] L. E. Dickson, History of the Theory of Numbers. Vol. II: Diophantine Analysis, Chelsea, New York, 1966, xxv+803.

[Gy] K. Győry, Power values of products of consecutive integers and binomial coeffcients, in: Number Theory and its Applications, Kluwer, 1999, 145-156.

[GyHS] K. Györy, L. Hajdu and N. Saradha, On the diophantine equation $n(n+d) \ldots$ $(n+(k-1) d)=b y^{l}$, Canad. Math. Bull., to appear.

[M] L. J. Mordell, Diophantine Equations, Academic Press, London, 1969.

[SS] N. Saradha and T. N. Shorey, Contributions towards a conjecture of Erdös on perfect powers in arithmetic progression, J. Reine Angew. Math., submitted. 
[S1] T. N. Shorey, Exponential diophantine equations involving products of consecutive integers and related equations, in: Number Theory, R. P. Bambah, V. C. Dumir and R. J. Hans-Gill (eds.), Hindustan Book Agency, 1999, 463-495.

[S2] -, Powers in arithmetic progression, in: A Panorama of Number Theory, G. Wüstholz (ed.), Cambridge Univ. Press, Cambridge, 2002, 325-336.

[S3] - Powers in arithmetic progression (II), in: New Aspects of Analytic Number Theory, Kyoto, 2002, 202-214.

[T1] R. Tijdeman, Diophantine equations and diophantine approximations, in: Number Theory and Applications, R. A. Mollin (ed.), Kluwer, 1989, 215-243.

[T2] - Exponential diophantine equations 1986-1996, in: Number Theory, de Gruyter, 1998, 523-539.

[vdW] B. L. van der Waerden, Beweis einer Baudetschen Vermutung, Nieuw Arch. Wisk. 19 (1927), 212-216.

Number Theory Research Group

of the Hungarian Academy of Sciences

Institute of Mathematics

University of Debrecen

P.O. Box 12

4010 Debrecen, Hungary

E-mail: hajdul@math.klte.hu

Received on 14.8.2003

and in revised form on 1.3.2004 